\title{
Ecoanatomía de la madera de dos taxa mexicanos del género Mimosa (Leguminosae-Mimosoideae)
}

\author{
Ecological anatomy of the wood of two Mexican taxa of the genus \\ Mimosa (Leguminosae-Mimosoideae)
}

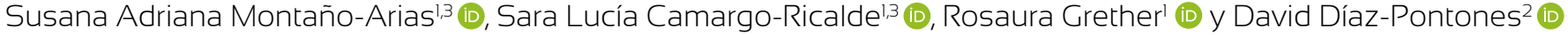

1 Universidad Autónoma Metropolitana-Iztapalapa, Departamento de Biología, División de Ciencias Biológicas y de la Salud, Apdo. Postal 55-535, 09340 CDMX, México.

2 Universidad Autónoma Metropolitana-Iztapalapa, Departamento de Ciencias de la Salud, División de Ciencias Biológicas y de la Salud, Apdo. postal 55-535 09340, CDMX, México.

3 Autores para la correspondencia: arias_susan@hotmail.com, slcr@xanum.uam.mx

\begin{abstract}
Citar como:
Montaño-Arias, S. A., S. L. CamargoRicalde, R. Grether y D. Díaz-Pontones. 2017. Ecoanatomía de la madera de dos taxa mexicanos del género Mimosa (Leguminosae-Mimosoideae). Acta Botanica Mexicana 118: 105120. DOl: http://dx.doi.org/10.21829/ abm118.2017.1203
\end{abstract}

Recibido: 2 de diciembre de 2015 Revisado: 4 de julio de 2016. Aceptado: 10 de octubre de 2016.

DOI:

http://dx.doi.org/10.21829/abml18. 2017.1203

\section{Resumen:}

Antecedentes y Objetivos: Los estudios ecoanatómicos permiten estimar la respuesta de los taxa a distintos factores ambientales. Considerando la relevancia biológica y ecológica del género Mimosa, se estimaron las posibles respuestas adaptativas de dos taxa endémicos de México, M. aculeaticarpa var. aculeaticarpa y M. luisana, a través de un análisis ecoanatómico de su madera, para evaluar su resistencia y tolerancia a cambios en la precipitación.

Métodos: Se recolectaron tres árboles de talla y cobertura similar por taxon. Se cortaron cubos de madera de $1 \times 1 \mathrm{~cm}$, ablandados a reflujo. Se hicieron secciones transversales, tangenciales y radiales, con grosor de 20 $\mu \mathrm{m}$. Se tiñeron con safranina-verde rápido y se montaron en resina sintética. La descripción y el análisis de los caracteres anatómicos de la madera se basaron en la nomenclatura propuesta por la IAWA.

Resultados clave: Mimosa aculeaticarpa var. aculeaticarpa presenta porosidad anular, mientras que $M$. luisana tiene porosidad difusa. Mimosa aculeaticarpa var. aculeaticarpa muestra vasos con diámetro tangencial mediano en madera temprana y pequeño en madera tardía y $M$. luisana presenta vasos con diámetro tangencial pequeño. Ambos tienen placa de perforación simple, punteaduras intervasculares areoladas, alternas y pequeñas, y ornamentadas en $M$. luisana. Mimosa aculeaticarpa var. aculeaticarpa posee principalmente parénquima axial paratraqueal vasicéntrico, mientras que en $M$. luisana es vasicéntrico y unilateral. En M. aculeaticarpa var. aculeaticarpa, los radios son uniseriados y en M. luisana biseriados. Las fibras son de longitud corta, diámetro fino y pared gruesa. Los índices de vulnerabilidad (IV) y de mesomorfía (IM) más altos se encontraron en M. aculeaticarpa var. aculeaticarpa (4.05 y 729.05, respectivamente), lo que indica que este taxon es más vulnerable a la sequía, mientras que los valores más bajos de IV y de IM correspondieron a M. luisana (1.42 y 226.71, respectivamente), sugiriendo que éste es más resistente a la sequía.

Conclusiones: Se propone que M. aculeaticarpa var. aculeaticarpa es apta para restaurar lugares mésicos y M. luisana lo es para ambientes secos.

Palabras clave: ambiente árido, ambiente mésico, estrés hídrico, Leguminosae, México, restauración.

\section{ABSTRACT:}

Background and Aims: Eco-anatomical studies enable the estimation of the response of taxa to different environmental factors. Considering the biological and ecological relevance of the genus Mimosa, the adaptive possible responses of two endemic Mexican taxa were estimated, M. aculeaticarpa var. aculeaticarpa y $M$. luisana, through the eco-anatomical analysis of their wood, in order to evaluate their resistance and tolerance to changes in precipitation.

Methods: Three trees of similar size and coverage per taxon, were collected. Wood cubes of $1 \times 1 \mathrm{~cm}$ were cut and softened by boiling. Transverse, tangential and radial sections, $20 \mu \mathrm{m}$ in thickness were made. Samples were stained with fast safranin-green and mounted in synthetic resin. The description and the analysis of anatomical wood features were based on the nomenclature proposed by the IAWA.

Key results: Mimosa aculeaticarpa var. aculeaticarpa presents ring-porosity, while M. luisana is characterized by diffuse porosity. Mimosa aculeaticarpa var. aculeaticarpa shows vessels with medium tangential diameter in early-wood, and small ones in late-wood, whereas M. luisana presents vessels with small tangential diameter. Both have simple perforation plates, bordered, alternate and small intervessel pits, and vestured ones in M. luisana. Mimosa aculeaticarpa var. aculeaticarpa mainly has axial paratracheal vasicentric parenchyma, while in M. luisana, it is vasicentric and unilateral. In M. aculeaticarpa var. aculeaticarpa, rays are uniseriate, and in M. luisana, they are biseriate. Fibers are of short length, fine diameter and thick wall. The highest indexes of vulnerability (VI) and of mesomorphy (MI) were found in M. aculeaticarpa var. aculeaticarpa (4.05 y 729.05, respectively), indicating that this taxon is the most vulnerable to drought, while the lowest values of both VI and MI corresponded to M. luisana (1.42 and 226.71 , respectively), suggesting that this taxon is more resistant to drought.

Conclusions: It is proposed that M. aculeaticarpa var. aculeaticarpa is suitable for restoring mesic sites and M. luisana for dry environments.

Key words: arid environments, Leguminosae, mesic environments, Mexico, restoration, water stress. 


\section{INTRODUCCIÓN}

Los estudios ecoanatómicos permiten analizar la estructura interna de las plantas con el fin de conocer su variación frente a los cambios ambientales y encontrar relaciones que pueden ser consideradas estrategias adaptativas (Araque y León, 2006; Polanco y Grande, 2009; Figueroa et al., 2011). Al respecto, se ha analizado el efecto de algunos parámetros ambientales como la disponibilidad de agua, estacionalidad, latitud, longitud y altitud sobre los caracteres anatómicos de la madera; por ejemplo, el diámetro de los vasos y el número de vasos por área se relaciona con la altitud (Baas, 1973; BarajasMorales, 1985; Fisher et al., 2007) y la longitud de los elementos de vaso con la disponibilidad de agua (Barajas-Morales, 1985; Lindorf, 1994; Parra, 2010; Giménez et al., 2012).

De acuerdo con Jono (2009), los estudios ecoanatómicos muestran la plasticidad de las plantas en la estructura de la madera, lo que aunado a otras características les permite sobrevivir en lugares con condiciones contrastantes, sobre todo, en especies de amplia distribución. Sin embargo, las variaciones ambientales ocurren a diferentes escalas y pueden influir en el crecimiento y, por consiguiente, en la anatomía de la madera (Luchi, 1998). En los últimos años, los estudios ecoanatómicos de la madera han adquirido relevancia, debido a que aportan información sobre el comportamiento de los taxa, y probablemente permitan predecir sus respuestas ante cambios ambientales (cambio climático). Este tipo de estudios han sido realizados, principalmente, en especies arbóreas, muchas de ellas pertenecientes a la familia Leguminosae (de Lima et al., 2009; Silva et al., 2011).

Las leguminosas son una de las cinco familias más diversas que existen en el mundo (Rzedowski, 1991; Sosa y Dávila, 1994). Asimismo, son uno de los elementos dominantes o co-dominantes de las zonas áridas y semiáridas mexicanas (de la Barrera y Andrade, 2005). Por su parte, Mimosa es el género más diverso de Mimosoideae en México, con ca. 110 especies distribuidas desde las zonas áridas y semiáridas hasta las zonas templadas (Grether et al., 1996).
A nivel mundial, se ha estudiado la anatomía de la madera de 43 especies de Mimosa, de un total de ca. 530 especies (Särkinen et al., 2011; Simon et al., 2011). Aunque en México existen ca. 68 taxa leñosos (Barneby, 1991), la madera de solo 12 de ellos ha sido estudiada. La mayoría de los trabajos están enfocados en describir la anatomía de la madera (Cozzo y Cristiani, 1950; Cozzo, 1951; Heringer y De Paula, 1979; Marchiori, 1982, 1985, 1993, 1996; Maccari y Marchiori, 1994; Marchiori y Muñiz, 1997) y, unos cuantos, en determinar los caracteres que pudiesen ayudar a resolver problemas de índole taxonómico dentro de la clasificación del género (BarettaKuipers, 1981; Chehaibar y Grether, 1990; MontañoArias, 2010). Más aún, la información que se encuentra en dichos estudios también es útil en estudios ecoanatómicos, ya que además de proporcionar información genética permiten asociar los caracteres anatómicos de los taxa con su hábitat (Montaño-Arias et al., 2011). Es decir, un enfoque ecoanatómico permite estimar la respuesta de los taxa a distintos factores ambientales (p. ej. precipitación); no obstante, estos estudios son escasos, particularmente en el género Mimosa (Montaño-Arias et al., 2013).

Por lo anterior y considerando la relevancia biológica y ecológica de algunas especies de Mimosa (CamargoRicalde et al., 2001; Camargo-Ricalde et al., 2002; Camargo-Ricalde et al., $2010 \mathrm{a}, \mathrm{b}$ ), el objetivo de este estudio es estimar las posibles respuestas adaptativas de dos taxa de Mimosa a nivel de la madera, con la finalidad de evaluar su resistencia y tolerancia a cambios en la precipitación.

\section{Materiales y MÉTOdOS}

\section{Método de colecta}

Se seleccionaron dos taxa arbóreos, endémicos de México, Mimosa aculeaticarpa Ortega var. aculeaticarpa y $M$. luisana Brandegee (Leguminosae) que crecen en condiciones ambientales contrastantes. De acuerdo con Grether et al. (2007), Mimosa aculeaticarpa var. aculeaticarpa presenta una amplia distribución en el país, estableciéndose tanto en climas secos como templados, mientras que M. luisana es un taxon restringido al Valle de Tehuacán- 
Cuicatlán, en Puebla y Oaxaca, y solo se establece en climas secos (Martínez-Bernal y Grether, 2006, Cuadro 1).

Para cada sitio de colecta se registraron las coordenadas geográficas, el tipo de vegetación (Rzedowski, 1978), clima (García, 2004) y datos de los individuos (altura y diámetro del tronco, Cuadro 1). Con la finalidad de tener representada la variabilidad de cada taxon, se recolectaron tres árboles de talla y cobertura similar de cada uno; las muestras fueron tomadas del tronco a $80 \mathrm{~cm}$ del suelo (Cuadro 1).

Para la observación de las características anatómicas se cortaron cubos de $1 \times 1 \mathrm{~cm}$, los cuales fueron ablandados durante $3 \mathrm{~h}$ a reflujo en agua destilada, tiempo necesario para poder seccionar la madera. Posteriormente, con un micrótomo de deslizamiento (American Optical 860, New York, EUA) se cortaron secciones transversales, tangenciales y radiales, con grosor de $20 \mu \mathrm{m}$, las cuales se tiñeron con safranina-verde rápido y se montaron en resina sintética (Johansen, 1940).

Asimismo, se colocaron astillas de la sección radial en un tubo de ensayo con una mezcla, a partes iguales, de ácido acético, ácido láctico, ácido nítrico, y glicerina (1:1:1:1), dejándolos incubar por 1-2 semanas. Una vez disociado el material, éste se lavó con agua destilada y se hicieron preparaciones temporales para cuantificar la longitud de los elementos de vaso, de las fibras y de las traqueidas vasicéntricas. Los ejemplares de referencia y las tablillas correspondientes están depositados en el Herbario Metropolitano (UAMIZ) de la Universidad Autónoma Metropolitana, Unidad Iztapalapa (Cuadro 1).

Para la descripción y el análisis de los caracteres anatómicos de la madera, se siguió la nomenclatura propuesta por la International Association of Wood Anatomists (IAWA, 1989). Se realizaron 25 mediciones para cada carácter de la anatomía de la madera, por individuo, por taxon. El número de vasos por superficie $\left(\mathrm{mm}^{2}\right)$ se contabilizó tomando en cuenta a los vasos solitarios y agrupados, y cada vaso que conforma a los vasos agrupados se contabilizó como un vaso solitario (IAWA, 1989). El grosor de la pared del elemento de vaso se midió en corte transversal; el diámetro de la punteadura intervascular y el diámetro del lumen de la punteadura fueron medidos en corte tangencial, siguiendo la clasificación propuesta por Montaño-Arias (2010). Para la longitud del cordón de parénquima, se contabilizó el número de células por cordón, y de manera adicional, se midió la longitud $(\mu \mathrm{m})$. Para ello, se utilizó la clasificación para longitud de fibras de la IAWA (1989). Las mediciones se realizaron utilizando un microscopio óptico (Zeiss Primo-Star, Alemania).

Para el diámetro de los vasos se siguió lo propuesto por la IAWA (1989) con algunas modificaciones. Para calcular el Índice de Vulnerabilidad (IV), fue utilizado

Cuadro 1: Estado, localización y altitud, tipo de vegetación, clima, temperatura y precipitación de los sitios de recolección de las especies de Mimosa L. estudiadas, así como hábito, altura y diámetro a la altura del pecho (DAP), número de registro de herbario y xiloteca (UAMIZ) de los individuos seleccionados.

\begin{tabular}{|c|c|c|c|c|c|c|}
\hline Taxa & Estado & $\begin{array}{c}\text { Coordenadas } \\
\text { Altitud (m s.n.m.) }\end{array}$ & $\begin{array}{c}\text { Tipo de } \\
\text { vegetación }\end{array}$ & $\begin{array}{c}\text { Clima } \\
\text { Temperatura (Media anual) } \\
\text { Precipitación (mm anuales) }\end{array}$ & $\begin{array}{c}\text { Hábito } \\
\text { Altura (m) } \\
\text { DAP cm por } \\
\text { individuo }\end{array}$ & $\begin{array}{c}\text { UAMIZ } \\
\text { No. registro } \\
\text { Ejemplar / Tablilla }\end{array}$ \\
\hline $\begin{array}{l}\text { M. aculeaticarpa var. } \\
\text { aculeaticarpa }\end{array}$ & Michoacán & $\begin{array}{l}19^{\circ} 58.218^{\prime} \mathrm{N} \\
101^{\circ} 31.664^{\prime} \mathrm{O}\end{array}$ & $\begin{array}{l}\text { Bosque de } \\
\text { pino-encino }\end{array}$ & $\begin{array}{l}\text { Templado Suhúmedo } \\
16.8^{\circ} \mathrm{C}\end{array}$ & $\begin{array}{c}\text { Árbol } \\
2.5,2 \text { y } 2\end{array}$ & $\begin{array}{c}71454,71455 \\
71456\end{array}$ \\
\hline & & 1140 & XCTOIIU & $\begin{array}{l}23.8^{\circ} \mathrm{C} \\
440.6 \\
\end{array}$ & $4,4.5$ y 4 & 216 \\
\hline
\end{tabular}


el diámetro de los vasos (dv) y el número de vasos $/ \mathrm{mm}^{2}$ (nv) (IV), de acuerdo con la fórmula IV=dv/nv. Los resultados con un valor inferior a 1 se interpretan como resistentes a la sequía (Carlquist, 1977). La longitud del elemento de vaso (lv) y el IV se emplearon para calcular el Índice de Mesomorfía (IM), donde IM=IV(lv). Un IM mayor de 200 indica que el xilema es mesomórfico y un IM menor o igual a 75 indica un xilema xerófito (Carlquist, 1977).

\section{Análisis estadísticos}

Con el objeto de evaluar la existencia de diferencias estadísticamente significativas entre los caracteres evaluados inter-específicamente, los datos se examinaron por medio de una $t$-Student $(\mathrm{P} \leq 0.05$; Sokal y Rohlf, 1995). Los análisis estadísticos se llevaron a cabo mediante el paquete estadístico NCSS (Hintze, 2001).

\section{Resultados}

La madera de Mimosa aculeaticarpa var. aculeaticarpa muestra porosidad anular y la de M. luisana porosidad difusa. Ambas maderas tienen anillos de crecimiento definidos. En M. aculeaticarpa var. aculeaticarpa están delimitados por parénquima axial marginal y vasos de mayor diámetro (Fig. 1A), mientras que en la madera de M. luisana, los anillos son más angostos y están delimitados por ocho hileras de fibras engrosadas (Fig. 1B).

Independientemente del tipo de porosidad, la madera de ambos taxa presenta vasos numerosos $(>16$ vasos $/ \mathrm{mm}^{2}$ ). Cabe señalar que la madera temprana de M. aculeaticarpa var. aculeaticarpa tiene más vasos/ $\mathrm{mm}^{2}$ que la madera tardía (Cuadro 2). La madera de ambos taxa tiene vasos solitarios abundantes (Fig. 1A-B). Además, tanto la madera temprana como la tardía de $M$. aculeaticarpa var. aculeaticarpa presentan vasos agrupados en dos, rara vez se encuentran grupos de tres. En el caso de $M$. luisana, se observan principalmente tres vasos agrupados, pero se pueden llegar hasta seis (Fig. 1A-B).

\section{Elementos de vaso}

Los dos taxa tienen elementos de vaso con paredes delgadas, aunque el diámetro tangencial de los vasos difiere. En el caso de M. aculeaticarpa var. aculeaticarpa, la madera temprana tiene vasos de diámetro mediano y en la madera tardía son de diámetro pequeño; en M. luisana, solo hay

Cuadro 2: Características de los elementos de vaso de Mimosa aculeaticarpa Ortega var. aculeaticarpa y M. luisana Brandegee.

\begin{tabular}{|c|c|c|c|c|c|c|}
\hline \multicolumn{7}{|c|}{ Mimosa aculeaticarpa var. aculeaticarpa } \\
\hline \multirow[t]{2}{*}{ Variables } & \multicolumn{2}{|c|}{ Media \pm desviación estándar } & \multicolumn{2}{|c|}{ Valor mínimo } & \multicolumn{2}{|c|}{ Valor máximo } \\
\hline & Temprana & Tardía & Temprana & Tardía & Temprana & Tardía \\
\hline Vasos $/ \mathrm{mm}^{2}$ & $19.44 \pm 2.0$ & $16.77 \pm 6.7$ & 4 & 6 & 30 & 33 \\
\hline$\varnothing$ tangencial del vaso $(\mu \mathrm{m})$ & $106.26 \pm 5.1$ & $38.3 \pm 5.8$ & 55 & 22.5 & 152.5 & 57.5 \\
\hline Grosor de la pared de los elementos de vaso $(\mu \mathrm{m})$ & $4.53 \pm 1.2$ & $2.83 \pm 0.57$ & 3.3 & 2.5 & 6.6 & 4.1 \\
\hline Longitud de los elementos vaso $(\mu \mathrm{m})$ & $167.5 \pm 6.5$ & $193.2 \pm 4.0$ & 100 & 100 & 237.5 & 250 \\
\hline \multicolumn{7}{|c|}{ Mimosa luisana } \\
\hline & \multicolumn{2}{|c|}{ Media \pm desviación estándar } & \multicolumn{2}{|c|}{ Valor mínimo } & \multicolumn{2}{|c|}{ Valor máximo } \\
\hline Vasos $/ \mathrm{mm}^{2}$ & \multicolumn{2}{|c|}{$35.5 \pm 2.63$} & \multicolumn{2}{|c|}{22} & \multicolumn{2}{|c|}{49} \\
\hline$\varnothing$ tangencial del vaso $(\mu \mathrm{m})$ & \multicolumn{2}{|c|}{$50.3 \pm 2.9$} & \multicolumn{2}{|c|}{25} & \multicolumn{2}{|c|}{70} \\
\hline Grosor de la pared de los elementos de vaso $(\mu \mathrm{m})$ & \multicolumn{2}{|c|}{$7.17 \pm 0.61$} & \multicolumn{2}{|c|}{5} & \multicolumn{2}{|c|}{10} \\
\hline Longitud de los elementos vaso $(\mu \mathrm{m})$ & \multicolumn{2}{|c|}{$159.1 \pm 9.0$} & \multicolumn{2}{|c|}{50} & \multicolumn{2}{|c|}{250} \\
\hline
\end{tabular}




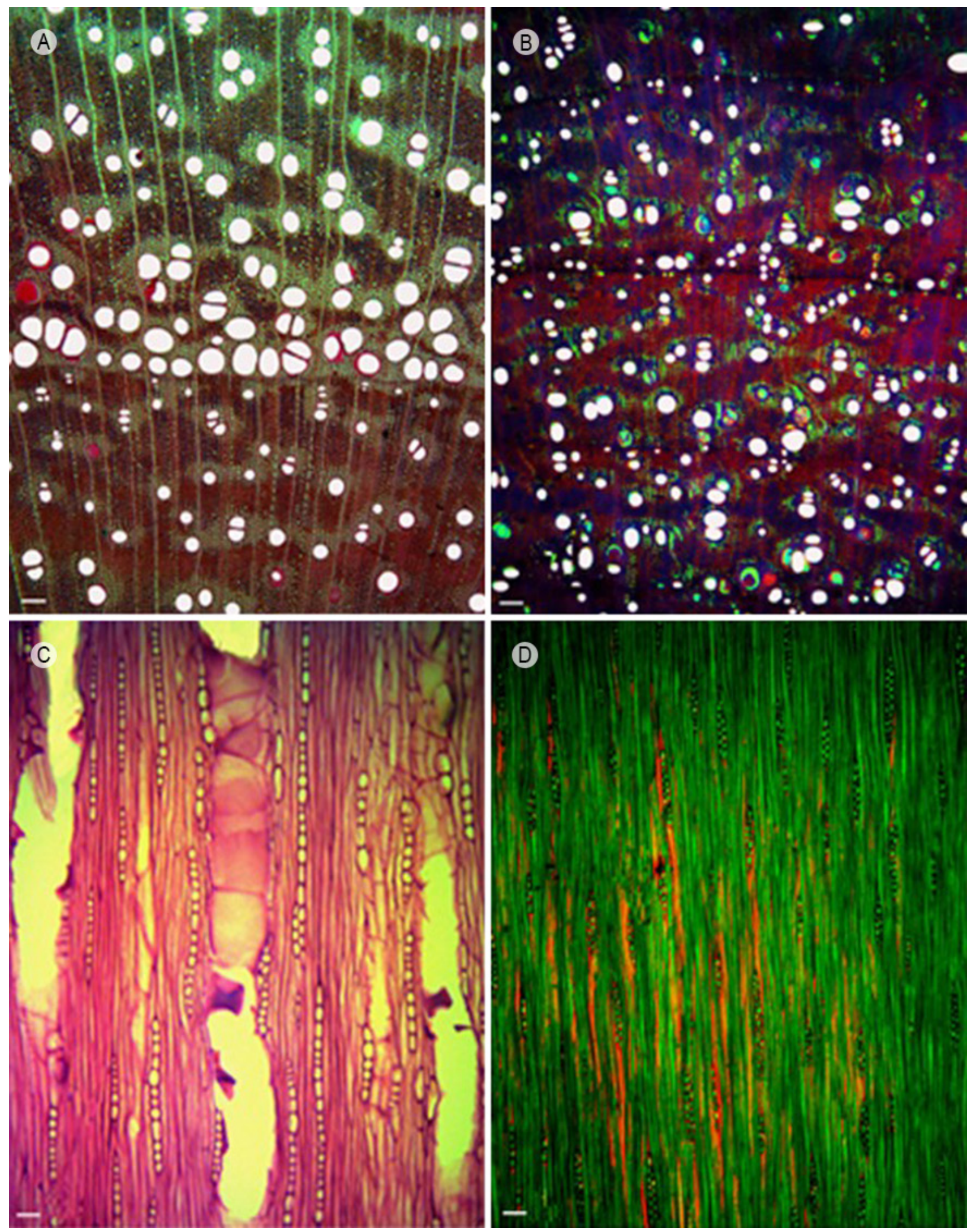

Figura 1: Cortes anatómicos de la madera de Mimosa aculeaticarpa Ortega var. aculeaticarpa y Mimosa luisana Brandegee. A. y C. M. aculeaticarpa; B. y D. M. luisana; A. B. cortes transversales; C. D. cortes tangenciales. Escala: $100 \mu \mathrm{m}$. 
de diámetro pequeño (Cuadro 2). Asimismo ambos presentan elementos de vaso cortos. Cabe señalar que en $M$. aculeaticarpa var. aculeaticarpa, los de la madera tardía son más largos que los de la madera temprana (Cuadro 2).

Ambos taxa tienen elementos de vaso con placa de perforación simple, punteaduras intervasculares alternas, areoladas, y en $M$. luisana, además son ornamentadas (Fig. 1C y Fig. 2A, C y D). Entre los taxa existen diferencias estadísticamente significativas con relación al diámetro de la punteadura intervascular y del lumen de la punteadura, siendo M. luisana la que mostró el mayor diámetro en ambos casos (Cuadro 3).

\section{Parénquima axial}

En ambos taxa se observó parénquima axial paratraqueal abundante y apotraqueal difuso. En el caso de M. aculeati- carpa var. aculeaticarpa, la madera temprana posee esencialmente parénquima aliforme, pero también presenta aliforme confluente, que une de dos a cuatro vasos, seguido de parénquima vasicéntrico y unilateral. En la madera tardía abunda el parénquima vasicéntrico, aliforme confluente, que une de dos a seis vasos, aliforme y unilateral (Fig. 1A).

El parénquima en la madera de M. luisana, primariamente, es aliforme confluente, que une de dos a ocho vasos, de manera que es un parénquima en bandas con más de tres células de ancho, seguido de aliforme, vasicéntrico y unilateral (Fig. 1B). En los dos taxa, las células del parénquima axial forman cordones cortos (Fig. 1C y D); sin embargo, M. aculeaticarpa var. aculeaticarpa presenta los cordones más largos, mostrando diferencias estadísticamente significativas con los de M. luisana (Cuadro 3). En el caso de M. aculeaticarpa var. aculeaticarpa, los cordones están

Cuadro 3: Estadística descriptiva y $t$-Student correspondientes a: punteaduras, parénquima axial, radios, fibras y traqueidas vasicéntricas de Mimosa aculeaticarpa Ortega var. aculeaticarpa y M. luisana Brandegee. Los valores seguidos con la misma letra indica que no hubo diferencia significativa, con $\mathrm{P}<0.05$. $\varnothing=$ Diámetro, ns=no significativo.

\begin{tabular}{|c|c|c|c|c|c|c|}
\hline \multirow[t]{2}{*}{ Variables } & \multirow[t]{2}{*}{ Taxa } & \multirow[t]{2}{*}{$\begin{array}{l}\text { Media } \pm \text { desviación } \\
\text { estándar }\end{array}$} & \multirow[t]{2}{*}{$\begin{array}{l}\text { Valor } \\
\text { mínimo }\end{array}$} & \multirow[t]{2}{*}{$\begin{array}{l}\text { Valor } \\
\text { máximo }\end{array}$} & \multicolumn{2}{|c|}{$\begin{array}{l}\text { t-Student } \\
(\mathrm{P} \leq 0.05)\end{array}$} \\
\hline & & & & & $\mathrm{t}$ & $\mathrm{P}$ \\
\hline \multirow{2}{*}{$\begin{array}{l}\text { Ø de la punteadura } \\
\text { intervascular }(\mu \mathrm{m})\end{array}$} & M. aculeaticarpa var. aculeaticarpa & $4.76 \pm 0.10^{\mathrm{b}}$ & 3.12 & 7.05 & 4.9680 & 0.007662 \\
\hline & M. luisana & $6.2 \pm 0.50^{\mathrm{a}}$ & 5 & 7.5 & & \\
\hline \multirow{2}{*}{$\begin{array}{l}\varnothing \text { del lumen de la punteadura } \\
\text { intervascular }(\mu \mathrm{m})\end{array}$} & M. aculeaticarpa var. aculeaticarpa & $2.76 \pm 0.3^{\mathrm{b}}$ & 2.02 & 3.42 & 3.3288 & 0.029139 \\
\hline & M. luisana & $3.73 \pm 0.50^{\mathrm{a}}$ & 2.5 & 5.0 & & \\
\hline \multirow{2}{*}{$\begin{array}{l}\text { Longitud del cordón de } \\
\text { parénquima }(\mu \mathrm{m})\end{array}$} & M. aculeaticarpa var. aculeaticarpa & $279.08 \pm 20.87^{\mathrm{a}}$ & 123.4 & 462.74 & 8.8392 & 0.000904 \\
\hline & M. luisana & $164.93 \pm 8.06^{\mathrm{b}}$ & 115 & 235 & & \\
\hline \multirow[t]{2}{*}{ Radios/mm } & M. aculeaticarpa var. aculeaticarpa & $6.71 \pm 0.68^{\mathrm{a}}$ & 4 & 10 & 2.9675 & 0.041245 \\
\hline & M. luisana & $5.20 \pm 0.32^{\mathrm{b}}$ & 3 & 8 & & \\
\hline \multirow[t]{2}{*}{ Altura de radios $(\mu \mathrm{m})$} & M. aculeaticarpa var. aculeaticarpa & $236.67 \pm 39.73^{\mathrm{a}}$ & 90 & 540 & 5.4473 & 0.005516 \\
\hline & M. luisana & $107.67 \pm 10.19^{\mathrm{b}}$ & 55 & 230 & & \\
\hline \multirow[t]{2}{*}{ Anchura de radios $(\mu \mathrm{m})$} & M. aculeaticarpa var. aculeaticarpa & $17.87 \pm 3.15$ & 10 & 35 & 0.5346 & $0.621278^{\mathrm{ns}}$ \\
\hline & M. luisana & $19.20 \pm 2.96$ & 10 & 25 & & \\
\hline \multirow[t]{2}{*}{ Longitud de fibras $(\mu \mathrm{m})$} & M. aculeaticarpa var. aculeaticarpa & $674.0 \pm 8.06$ & 553.33 & 830 & 0.7726 & $0.482864^{\mathrm{ns}}$ \\
\hline & M. luisana & $661.73 \pm 31.14$ & 480 & 890 & & \\
\hline \multirow[t]{2}{*}{$\varnothing$ del lumen de la fibra $(\mu \mathrm{m})$} & M. aculeaticarpa var. aculeaticarpa & $7.83 \pm 0.11$ & 5 & 5 & 2.0612 & $0.108304^{\mathrm{ns}}$ \\
\hline & M. luisana & $6.0 \pm 0.16$ & 10 & 17.5 & & \\
\hline \multirow{2}{*}{$\begin{array}{l}\text { Grosor de la pared de la fibra } \\
(\mu \mathrm{m})\end{array}$} & M. aculeaticarpa var. aculeaticarpa & $5.83 \pm 0.36$ & 3.33 & 10 & 1.1329 & $0.320575^{\text {ns }}$ \\
\hline & M. luisana & $5.0 \pm 0$ & 5 & 5 & & \\
\hline \multirow{2}{*}{$\begin{array}{l}\text { Longitud de traqueidas } \\
\text { vasicéntricas }(\mu \mathrm{m})\end{array}$} & M. aculeaticarpa var. aculeaticarpa & $668.27 \pm 8.81$ & 546.67 & 800 & 0.7990 & $0.469026^{\mathrm{ns}}$ \\
\hline & M. luisana & $659.33 \pm 26.7$ & 486.67 & 890 & & \\
\hline
\end{tabular}



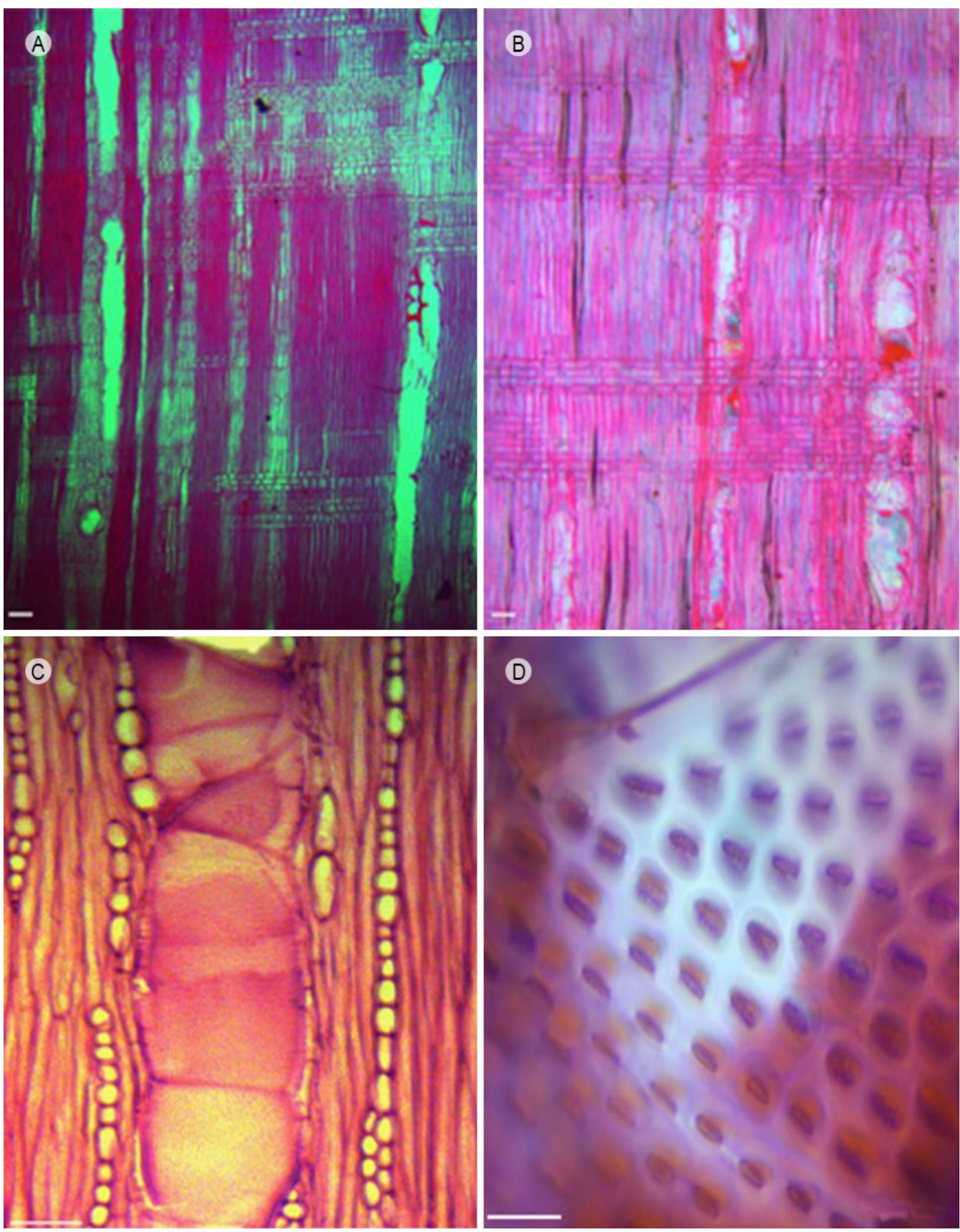

Figura 2: Cortes anatómicos de la madera de A. y C. Mimosa aculeaticarpa Ortega var. aculeaticarpa, y B. y D. Mimosa luisana Brandegee. A. y B. cortes radiales; C. y D. cortes tangenciales, punteaduras areoladas, alternas y ornamentadas. Escala: $10 \mu \mathrm{m}$. 
constituidos por una célula, rara vez se observan dos (Fig. 1C), mientras que en $M$. luisana están integrados por series de dos células y, rara vez, se observan una o tres células (Fig. 1D). Cabe mencionar que, únicamente las células del parénquima de $M$. luisana presentan de dos a 14 cristales prismáticos, cada uno de ellos ubicado en una cámara.

\section{Radios y fibras}

Los dos taxa presentan radios numerosos, pero existen diferencias significativas entre ellos, siendo M. aculeaticarpa var. aculeaticarpa la que presenta el mayor número de radios/mm y estos son esencialmente uniseriados, aunque hay biseriados (Fig. 1C). En M. luisana los radios son principalmente biseriados, pero también existen uniseriados (Fig. 1D). En ambos taxa, los radios son homocelulares, bajos y finos y están compuestos por células procumbentes (Fig. 2A y B). Estadísticamente, M. aculeaticarpa var. aculeaticarpa presenta los radios más altos (Cuadro 3). Por otro lado, las fibras son de longitud corta, diámetro fino y pared delgada. Además, presentan traqueidas vasicéntricas de longitud corta; sin embargo, en M. aculeaticarpa var. aculeaticarpa solo se encuentran en la madera temprana (Cuadro 3).

\section{Índices de Vulnerabilidad y Mesomorfía}

El IV muestra diferencias estadísticamente significativas entre los taxa ( $\mathrm{t}=7.0560 ; \mathrm{P}=0.002128)$. El valor más alto corresponde a M. aculeaticarpa var. aculeaticarpa (4.05), lo que indica que es más vulnerable a la sequía. Además, presenta el IM superior (729.05), mientras que los valores más bajos, tanto de IV como de IM, corresponden a $M$. luisana (1.42 y 226.71, respectivamente), lo que sugiere que esta especie es más resistente a la sequía. No obstante, debido a que ambos taxa presentan un IM superior a 200, se considera que presentan un xilema mesomórfico.

\section{DIsCUSIÓN}

De manera general, las características anatómicas de la madera observadas en ambos taxa de Mimosa son similares a las ya reportadas para la subfamilia Mimosoideae (Evans et al., 2006) y, en específico, para el género Mimo- sa (Heringer y De Paula, 1979; Marchiori, 1982; 1985; 1996; Maccari y Marchiori, 1994; Montaño-Arias, 2010). No obstante, el análisis en conjunto de trabajos previos sobre este tema y de los resultados obtenidos en este estudio muestra diferencias a nivel específico, en cuanto al tipo de porosidad, diámetro y longitud de los elementos vasculares (p. ej. vasos, traqueidas vasicéntricas) y altura de los radios, entre otros, por lo que es posible considerar que estos caracteres están influenciados por el ambiente (Woodcock, 1994; Wodzicki, 2001; León, 2005; Montaño-Arias et al., 2011). En cambio, otros caracteres como la predominancia de algún tipo de parénquima y el tipo de placas de perforación, son caracteres que, de acuerdo con Polanco y Grande (2009), están relacionados con los procesos evolutivos de las plantas.

\section{Porosidad}

La porosidad, ya sea anular o difusa, ha sido asociada con la disponibilidad de agua (Gilbert, 1940 en Moglia y Giménez, 1998; Bissing, 1982). La porosidad anular observada en M. aculeaticarpa var. aculeaticarpa también ha sido encontrada en otros taxa de Mimosa, como M. acantholoba (H. \& B. ex Willd.) Poir. var. eurycarpa (B.L. Rob.) Barneby, M. benthamii J.F. Macbr. var. benthamii, M. lacerata Rose y M. texana (A. Gray) Small var. filipes (Britton \& Rose) Barneby (MontañoArias, 2010). Sin embargo, es relevante señalar que la muestra de madera de M. aculeaticarpa var. aculeaticarpa proviene de un clima cálido-húmedo, con estacionalidad marcada, mientras que las de M. acantholoba var. eurycarpa, M. benthamii var. benthamii, M. lacerata y M. texana var. filipes provienen de climas semisecos, igualmente con estacionalidad marcada (MontañoArias, 2010).

De acuerdo con Gutiérrez (2009), el hecho de que haya una estacionalidad marcada favorece el desarrollo de una porosidad anular y, por lo tanto, la diferenciación de madera temprana y madera tardía. Según Woodcock (1994), el significado funcional de la porosidad anular deriva en una conducción rápida en la madera temprana, a través de los vasos de mayor diámetro, y la madera tardía 
garantiza la seguridad de la columna de agua mediante sus vasos pequeños. No obstante, en especies de zonas templadas, es más común observar maderas con porosidad anular que en especies de regiones áridas o semiáridas (Moglia y Giménez, 1998). Lo anterior concuerda con los resultados obtenidos, ya que $M$. aculeaticarpa var. aculeaticarpa (porosidad anular) fue colectada en una zona templada subhúmeda en bosque de pino-encino, mientras que $M$. luisana (porosidad difusa) fue colectada en una zona semiárida, en matorral xerófilo.

Además, a nivel mundial se ha estudiado la anatomía de la madera de 43 especies de Mimosa, de las ca. 530 existentes (Särkinen et al., 2011; Simon et al., 2011). De estos $43,74 \%$ presenta porosidad difusa, $21 \%$ tiene porosidad anular y únicamente $5 \%$, porosidad semianular. Al respecto, Gilbert (1940 en Moglia y Giménez, 1998) considera la porosidad difusa como una ventaja para transportar la escasa cantidad de agua existente en el hábitat. Con base en lo anterior, la predominancia de los taxa con porosidad difusa en ecosistemas secos también explica, entre otros factores, por qué el género Mimosa es uno de los elementos dominantes o co-dominantes en estos ambientes.

\section{Elementos de vaso}

Ambos taxa presentan vasos numerosos, característica común no solo de las leguminosas que habitan en sitios secos (Silva et al., 1989; Moglia y Giménez, 1998), sino del género Mimosa (Montaño-Arias, 2010; Montaño-Arias et al., 2013). Aunque los vasos numerosos son considerados como característicos de las especies xerófitas (Carlquist y Hoekman, 1985), también se han reportado en taxa de climas fríos (Miller, 1975), ya que en este tipo de ambientes las plantas incluso sufren de estrés hídrico (agua en forma de hielo o nieve). De acuerdo con León (2001), un mayor número de vasos está relacionado con el clima al darse un incremento de la sequía o una disminución de la temperatura del sitio donde se desarrolla la planta. Este dato es consistente con los resultados obtenidos, ya que M. luisana presentó el mayor número de vasos $/ \mathrm{mm}^{2}$, siendo un taxon que habita en un sitio cálido seco. Esta tendencia también ha sido observada en otras especies del género, como M. cruenta Benth., M. daleoides Benth., M. leucaenoides Benth., e incluso en otras mimosoideas como Acacia macracatha Benth., Calliandra gracilis Klotzsch (ahora Zapoteca formosa (Kunth) H.M.Hern. subsp. gracilis (Griseb.) H.M.Hern., Leucaena trichodes Benth. y Pithecellobium saman (Jacq.) Benth. (ahora Samanea saman (Jacq.) Merril) que habitan zonas secas (Silva et al., 1989).

Asimismo, los vasos de la madera temprana de $M$. aculeaticarpa var. aculeaticarpa presentan diámetros medianos, al igual que los de la madera de M. tenuiflo$r a$ (Willd.) Poir. (Montaño-Arias, 2010). De acuerdo con Carlquist (2001), esto favorece el manejo de grandes cantidades de agua por unidad de tiempo por vaso. Lo anterior sugiere que $M$. aculeaticarpa var. aculeaticarpa está adaptada para manejar mayores volúmenes de agua (estación de lluvias), en comparación con los vasos de diámetro pequeño de la madera tardía (estación de secas). En el caso de la madera de M. luisana, los vasos son más pequeños, indicando una mayor resistencia a la cavitación $\mathrm{y}$, por consiguiente, éstos contribuyen a reducir los embolismos (Polanco y Grande, 2009; Giménez et al., 2012) y muestran resistencia al estrés hídrico (Montaño-Arias et al., 2013).

La presencia de vasos agrupados es una ventaja, ya que confieren una mayor seguridad en la conducción de agua (Baas y Carlquist, 1985; Carlquist y Hoekman, 1985; Barajas-Morales, 1985), disminuyendo el riesgo por embolia (Moglia y Giménez, 1998). En este estudio, los resultados muestran que ambos taxa tienen vasos agrupados; sin embargo, M. luisana los presenta en mayor número, sugiriendo que su madera es la que provee mayor seguridad a la columna de agua.

Las paredes delgadas de los elementos de vaso han sido asociadas a lugares húmedos (Montaño-Arias et al., 2013), lo que coincide con la madera de M. aculeaticarpa var. aculeaticarpa, que crece en un clima subhúmedo. Aunque en M. luisana los elementos de vaso también tienen paredes delgadas, su grosor es aproximadamente del doble de un vaso de la madera temprana y tres veces 
el grosor de la pared de un vaso de la madera tardía de M. aculeaticarpa var. aculeaticarpa.

\section{Longitud de elementos de vaso, placas de perforación y punteaduras intervasculares}

La longitud de los elementos de vaso es un rasgo de adaptación evolutiva, ya que a medida que éstos sean más cortos (Zimmermann, 1978) y anchos, habrá mayor seguridad en la conducción del agua. Los resultados muestran que ambos taxa tienen elementos de vaso cortos, por lo que ambos proveen seguridad en el proceso conductivo. Además, los resultados son consistentes con lo reportado por Carlquist y Hoekman (1985), en cuanto a que la longitud de los elementos de vaso disminuye con la aridez, ya que M. luisana tiene los elementos de vaso más cortos y se ubica en un hábitat con menor humedad que el ambiente en donde se establece de M. aculeaticarpa var. aculeaticarpa. Esta tendencia también ha sido observada en $M$. leucaenoides que presenta los elementos de vaso más cortos que los taxa estudiados (Montaño-Arias et al., 2013).

De acuerdo con Carlquist $(1975,2001)$ y MontañoArias et al. (2013), los elementos de vaso de longitud corta son considerados los más fuertes, a causa del estrechamiento formado por la pared del elemento de vaso, aún en aquellos que presentan placa de perforación simple, ya que esto permite resistir grandes presiones y deformaciones relacionadas con las tensiones de la columna de agua.

La presencia de placas de perforación simple implica una óptima conducción (Moglia y López, 2001) genera un aumento en la conductividad hidráulica, pero produce una mayor vulnerabilidad a los embolismos (Polanco y Grande, 2009). Sin embargo, esta vulnerabilidad es reducida cuando las placas de perforación simple están acompañadas por punteaduras intervasculares alternas y, más aún, cuando éstas son ornamentadas, ya que proporcionan tensión superficial mayor y disminuyen la propagación del émbolo (Jansen et al., 2003; 2004). Por un lado, las punteaduras intervasculares permiten el flujo de agua a los vasos adyacentes (Zweypfenning, 1978) $y$, por otro, la ornamentación retiene las burbujas de aire provocadas por una pérdida en la presión del agua (Carlquist, 1982; Choat et al., 2004) y, de esta forma, evitan la cavitación (Silva et al., 2011). En este estudio, únicamente $M$. luisana presentó punteaduras intervasculares ornamentadas. Cabe mencionar que este tipo de punteaduras también han sido reportadas en otros 17 taxa de Mimosa (p. ej. M. bimucronata (DC.) Kuntze, Cozzo, 1951; M. eriocarpa Benth., Carnieletto y Marchiori, 1993; M. incana (Spreng.) Benth., Marchiori, 1996; M. micropteris Benth., da Silva-Pereira et al., 2013; M. pilulifera Benth., Marchiori y Muñiz, 1997, y M. sparsa Benth., Maccari y Marchiori, 1994).

\section{Parénquima axial, radios y fibras}

De acuerdo con Aguilar-Rodríguez y Barajas-Morales (2005), el parénquima axial es un carácter relacionado con aspectos anatómico-evolutivos de las angiospermas, considerando que el parénquima aliforme y el aliforme confluente son los más evolucionados (Polanco y Grande, 2009). Con base en lo anterior, se sugiere que M. luisana es un taxon más reciente, ya que en su madera predominan ambos tipos de parénquima; sin embargo, esto debe fortalecerse con la realización de estudios de tipo ecofiléticos.

Aunque Carlquist (2001) y León (2001) han señalado que la altura de los radios posee una baja influencia ambiental, varios autores señalan lo contrario. Por ejemplo, Barajas-Morales (1985) reporta que la presencia de radios altos está asociada a especies que habitan en sitios mésicos, lo que coincide con los resultados obtenidos, ya que M. aculeaticarpa var. aculeaticarpa crece en este tipo de sitios y presenta radios más altos que M. luisana.

Por su parte, Novaes et al. (2010) mencionan que existe una relación directa entre la altura de los radios y la disponibilidad de agua en el sitio, de modo que los radios bajos implican que, en el sitio, existe poca disponibilidad de agua. Aún más, Alves y Angyalossy-Alfonso (2002) y Amano (2007) señalan que, además de la altura de los radios, el grosor de la pared de las fibras, también está relacionado con la disponibilidad de agua. Estos autores reportan que las fibras con pared gruesa son frecuentes en 
especies de zonas áridas. Sin embargo, los dos taxa aquí estudiados, así como $M$. detinens Benth., M. adpressa $\mathrm{H}$. et A., M. ostenii Speg. ex Burk., M. uliginosa Chod. et Hassl. y M. scabrella Benth. (Cozzo, 1951), entre otras, presentan fibras de paredes delgadas, independientemente del sitio en el que se desarrollan, lo que no coincide con lo reportado por estos autores. En forma adicional, de acuerdo con Polanco y Grande (2009), los resultados sugieren que ambos taxa tienen, en términos anatómicoevolutivos, una madera avanzada por presentar elementos de vaso con placa de perforación simple, asociados a fibras libriformes.

Por otro lado, las fibras se asocian con un alto número de vasos solitarios, lo que sugiere que los taxa están establecidos en un hábitat con alto grado de aridez. Esto permite entender por qué ambos tienen traqueidas vasicéntricas como un elemento conductor adicional orientado hacia la seguridad, que permita transportar la escasa cantidad de agua existente en su hábitat. Mimosa aculeaticarpa var. aculeaticarpa presenta traqueidas vasicéntricas únicamente en la madera temprana, lo que indica que, durante la estación de lluvias, requiere de elementos conductores adicionales que le permitan transportar el agua. Además, la presencia de este carácter les confiere a ambos taxa una mayor seguridad en la conducción de agua (Montaño-Arias et al., 2013), volviéndolas más competitivas, ya que las traqueidas vasicéntricas funcionan como un sistema de conducción subsidiario o alternativo que cumple la misma función que los vasos agrupados (Moglia y Giménez, 1998).

\section{Índices de Vulnerabilidad y Mesomorfía}

Ambos taxa presentan un alto número de vasos reflejado en un IV superior a la unidad, por lo que se considera que son vulnerables a la sequía. Sin embargo, el IV que muestra la madera de M. aculeaticarpa var. aculeaticarpa supera casi tres veces el IV de M. luisana, lo que indica que esta última es la más resistente a la sequía.

Los IV son similares a los reportados en M. tenuiflora (6.26), M. tejupilcana R. Grether \& A. MartínezBernal (4.09), M. hexandra Micheli. (3.56), M. bahamen- sis Benth. (3.36) y M. leucaenoides (1.81), y coinciden en que son taxa vulnerables pero con un xilema adaptado para resistir la sequía (Montaño-Arias et al., 2013).

Respecto al IM, los taxa estudiados presentaron un xilema de tipo mesomórfico. Este tipo de xilema ya ha sido reportado para M. bahamensis, M. hexandra, M. leucaenoides, M. tejupilcana y M. tenuiflora. Sin embargo, el valor de IM de M. luisana es más parecido con M. leucaenoides (276.77) debido a que son taxa que habitan en ambientes secos. El valor de IM de M. aculeaticarpa var. aculeaticarpa se parece al de M. tejupilcana (727.76), ya que ambas crecen en ambientes subhúmedos. El xilema mesomórfico en conjunto con valores de IV menores a cinco sugiere que los taxa estudiados tienen un sistema de conducción seguro y eficaz para el movimiento del agua (León, 2001, 2001-2002).

\section{Conclusiones}

Los taxa estudiados tienen un xilema adaptado para resistir períodos de escasez de agua que les permite vivir y adaptarse a los ambientes en los que habitan. Se reconoce que la existencia de elementos de vaso de diámetro tangencial pequeño, longitud corta, con placa de perforación simple, punteaduras intervasculares ornamentadas y la presencia de traqueidas vasicéntricas contribuyen a la seguridad en la conducción de agua y son caracteres distintivos de taxa con capacidad de adaptación a diferentes tipos de hábitats.

La estructura de la madera es solo una más de las múltiples estrategias adaptativas (p. ej. hojas pequeñas y plantas caducifolias) que tienen los taxa estudiados para resistir o tolerar los períodos de escasez de agua. Asimismo, la comparación de estos resultados, con relación a lo reportado para otros taxa del género Mimosa, revela que taxa, tanto de climas templados como secos, presentan estrategias adaptativas semejantes, lo que explica su capacidad de colonizar ambientes desfavorables; es decir, aquellos hábitats en los que existen condiciones extremas de temperatura y/o precipitación.

Es preciso mencionar que, a pesar de que se ha estudiado la anatomía de la madera de 43 especies de 
Mimosa, son pocos los trabajos que tienen un enfoque ecoanatómico y/o ecofisiológico (Barajas-Morales, 1985; Barajas-Morales y León-Gómez, 1989; Montaño-Arias et al., 2013), lo que pone de manifiesto la necesidad de avanzar en esta línea para una evaluación comparativa que permita sugerir los taxa idóneos para diferentes ambientes en México. Algunas de las descripciones anatómicas llegan a ser ambiguas, ya que en ellas no se mencionan algunos caracteres o no dan una explicación de la característica encontrada lo que impide llegar a una conclusión ecoanatómica que integre a todos los taxa estudiados hasta ahora. No obstante, los resultados aquí presentados son útiles para sugerir la factibilidad de la reforestación de ecosistemas secos y mésicos. Se propone a M. luisana para restaurar ambientes secos y a $M$. aculeaticarpa var. aculeaticarpa para lugares mésicos.

\section{CONTRIBUCIONES DE AUTORES}

SAMA, SLCR y RGG concibieron y diseñaron el estudio, trabajo de campo, el diseño y desarrollo experimental. SAMA, SLCR, RGG, en colaboración con DDP, realizaron el análisis e interpretación de los datos. SAMA redactó el manuscrito con ayuda de SLCR, RGG y DDP. Todos los autores contribuyeron con la discusión, revisión y aprobación del manuscrito final.

\section{FINANCIAMIENTO}

Este estudio fue apoyado por la Universidad Autónoma Metropolitana, Unidad Iztapalapa, proyecto "Biología y ecología del género Mimosa (Leguminosae-Mimosoideae) en ecosistemas áridos y semiáridos de México: el caso del Valle de Tehuacán-Cuicatlán como un sistema modelo. Fase 1." y por el Consejo Nacional de Ciencia y Tecnología (CONACYT) por la beca de doctorado (228993/211528) otorgada a SAMA.

\section{AgRADECIMIENTOS}

Al Doctorado en Ciencias Biológicas y de la Salud de la Universidad Autónoma Metropolitana y al Consejo Nacional de Ciencia y Tecnología (CONACYT).

\section{LITERATURA CITADA}

Aguilar-Rodríguez, S. y J. Barajas-Morales. 2005. Anatomía de la madera de especies arbóreas de un bosque mesófilo de montaña: un enfoque ecológico-evolutivo. Boletín de la Sociedad Botánica de México 77: 51-58.

Alves, E. S. y V. Angyalossy-Alfonso. 2002. Ecological trends in the wood anatomy of some Brazilian species. 2. Axial parenchyma, rays and fibers. The International Association of Woods Anatomists Journal 23(4): 391-418.

Amano, E. 2007. Pau-brasil, madeira e casca: formação, desenvolvimento e estrutura. Tese doutorado em ciências, área Botânica. Instituto de Biociências, Universidade de São Paulo. São Paulo, Brasil. 107 pp. DOI: http://dx.doi. org/10.11606/T.41.2007.tde-25102007-181719

Araque, A. y H. W. León. 2006. Anatomía comparada del leño de Spondias mombin L. (Anacardiaceae) que crece en zonas de banco y bajío en la Reserva Forestal Caparo (Barinas, Venezuela). Revista Forestal Venezolana 50(1): 9-17.

Baas, P. 1973. The wood anatomical range in Ilex (Aquifoliaceae) and its ecological and phylogenetic significance. Blumea 21: 193-258.

Baas, P. y S. Carlquist. 1985. A comparison of ecological wood anatomy of the floras of southern California and Israel. The International Association of Wood Anatomists Bulletin 6(4): 349353. DOI: http://dx.doi.org/10.1163/22941932-90000961

Barajas-Morales, J. 1985. Wood structural differences between trees of two tropical forests in Mexico. The International Association of Wood Anatomists Bulletin 6(4): 355-365. DOI: http://dx.doi.org/10.1163/22941932-90000962

Barajas-Morales, J. y C. León-Gómez. 1989. Anatomía de maderas de México: especies de una selva baja caducifolia. Publicaciones Especiales 1. Universidad Nacional Autónoma de México, Instituto de Biología. México, D.F., México. Pp. 79-80.

Baretta-Kuipers, T. 1981. Wood anatomy of Leguminosae: its relevance to taxonomy. In: Polhill, R. M. y P. H. Raven (eds.). Advances in Legume Systematics. Royal Botanic Gardens. Kew, UK. Pp. 677-705.

Barneby, R. C. 1991. Sensitivae Censitae. A description of the genus Mimosa L. (Mimosaceae) in the New World. Memoirs of The New York Botanical Garden 65: 1-835. 
Bissing, D. R. 1982. Variation in qualitative anatomical features of the xylem of selected dicotyledonous woods in relation to water availability. The Bulletin of the Torrey Botanical Club 109: 371-384.

Camargo-Ricalde, S. L., R. Grether, A. Martínez-Bernal, V. García-García y S. Barrios-del-Rosal. 2001. Especies útiles del género Mimosa (Fabaceae-Mimosoideae) en México. Boletín de la Sociedad Botánica de México 68: 33-44.

Camargo-Ricalde, S. L., S. S. Dhillion y R. Grether. 2002. Community structure of endemic Mimosa species and environmental heterogeneity in a semi-arid Mexican valley. Journal of Vegetation Science 13(5): 697-704.

Camargo-Ricalde, S. L., I. Reyes-Jaramillo y N. M. Montaño. 2010a. Forestry insularity effect of four Mimosa L. species (Leguminosae-Mimosoideae) on soil nutrients of a Mexican semiarid ecosystem. Agroforest Systems 80: 385-397. DOI: http://dx.doi.org/10.1007/s10457-010-9330-y

Camargo-Ricalde, S. L., N. M. Montaño, I. Reyes-Jaramillo, C. Jiménez-González y S. S. Dhillion. 2010b. Effect of mycorrhizae on seedlings of six endemic Mimosa L. species (Leguminosae-Mimosoideae) from the semi-arid Tehuacán-Cuicatlán Valley, Mexico. Trees-Structure and Function 24: 67-78. DOI: http://dx.doi.org/10.1007/ s00468-009-0379-Z

Carlquist, S. 1975. Ecological strategies of xylem evolution. University of California Press. Berkeley, USA. 259 pp.

Carlquist, S. 1977. Ecological factors in wood evolution: a floristic approach. American Journal of Botany 64(7): 887896.

Carlquist, S. 1982. Wood anatomy of Onagraceae: further species; root anatomy; significance of vestured pits and allied structures in dicotyledons. Annals of the Missouri Botanical Garden 69(4): 755-769. DOI: http://dx.doi. org/10.2307/2398995

Carlquist, S. 2001. Comparative wood anatomy. Systematic, ecological and evolutionary aspects of dicotyledon wood. Springer Verlag. Berlín, Germany. 457 pp. DOI: http://dx.doi.org/10.1007/978-3-662-04578-7

Carlquist, S. y D. Hoekman. 1985. Ecological wood anatomy of the woody southern California Flora. The International Association of Wood Anatomists Bulletin 6(4): 319-347.
Carnieletto, C. y J. N. C. Marchiori. 1993. Anatomia da madeira de Mimosa eriocarpa Benth. Ciência Florestal 3(1): $107-$ 120.

Chehaibar, M. y R. Grether. 1990. Anatomía de la madera de algunas especies del género Mimosa (Leguminosae). Boletín de la Sociedad Botánica de México 50: 3-17.

Choat, B., S. Jansen, M. A. Zwieniecki, E. Smets y N. M. Holbrook. 2004. Changes in pit membrane porosity due to deflection and stretching: the role of vestured pits. Journal of Experimental Botany 55(402): 1569-1575. DOI: http:// dx.doi.org/10.1093/jxb/erh173

Cozzo, D. 1951. Anatomía del leño secundario de las Leguminosas Mimosoideas y Caesalpinoideas Argentinas, silvestres y cultivadas. Revista del Instituto Nacional de Investigación de las Ciencias Naturales y Museo Argentino de Ciencias Naturales Bernardino Rivadavia-Ciencias Biológicas 2(2): 63-146.

Cozzo, D. y L. Q. Cristiani. 1950. Los géneros de fanerógamas argentinas con estructura leñosa estratificada. Revista del Instituto Nacional de Investigación de las Ciencias Naturales y Museo Argentino de Ciencias Naturales Bernardino Rivadavia-Ciencias Biológicas 1: 363-405.

da Silva-Pereira, A. C., A. M. Siegloch y J. N. C. Marchiori. 2013. Anatomia do lenho de Mimosa micropteris Benth. Balduinia 40: 18-22.

de la Barrera, E. y A. Andrade. 2005. Challenges to plant megadiversity: how environmental physiology can help. New Phytologist 167(1): 5-8.

de Lima, R. S., P. L. De Oliveira y L. R. Rodrigues. 2009. Anatomia do lenho de Enterolobium contortisiliquum (Vell.) Morong. (Leguminosae-Mimosoideae) ocorrente em dois ambientes. Revista Brasileira de Botânica 32: 361-374. DOI: http://dx.doi.org/10.1590/S010084042009000200015

Evans, J. A., P. E. Gasson y G. P. Lewis. 2006. Wood anatomy of the Mimosoideae (Leguminosae). The International Association of Wood Anatomists Journal Supplement 5: $1-117$.

Figueroa, M. E., A. M. Giménez, P. Hernández, C. Gaillard de Benítez y G. Lorenz. 2011. Ecological wood anatomy of Allenrolfea vaginata (Griseb.) Kuntze (Chenopodiaceae) 
in saline environments in Santiago del Estero. Ecología Austral 21(3): 339-352.

Fisher, B. J., G. Goldstein, T. J. Jones y S. Cordell. 2007. Wood vessel diameter is related to elevation and genotype in the Hawaiian tree Metrosideros polymorpha (Myrtaceae). American Journal of Botany 94(5): 709-715. DOI: http:// dx.doi.org/10.3732/ajb.94.5.709

García, E. 2004. Modificaciones al Sistema de Clasificación climática de Köppen (para adaptarlo a las condiciones de la República Mexicana). Instituto de Geografía, Universidad Nacional Autónoma de México. México, D.F., México. 90 pp.

Giménez, A. M., F. Calatayu, M. E. Figueroa y J. Díaz-Zirpolo. 2012. Anatomía de leño de Maytenus vitis-idaea y M. viscifolia (Celastraceae). Boletín de la Sociedad Argentina de Botánica 47: 351-362.

Grether, R., S. L. Camargo-Ricalde y A. Martínez-Bernal. 1996. Especies del género Mimosa (Leguminosae) presentes en México. Boletín de la Sociedad Botánica de México 58: 149-152.

Grether, R., A. Martínez-Berna y S. L. Camargo-Ricalde. 2007. Mimosa. Flora del Bajío y de regiones adyacentes 150 : 140-183.

Gutiérrez, M. E. 2009. La dendrocronología: métodos y aplicaciones. In: Nieto, X. y M. A. Cau (eds.). Arqueologia nàutica mediterránia $\mathrm{CASC}$, Museo de Arqueología de Catalunya. Catalunya, España. Pp. 309-322.

Heringer, E. P. y J. E. de Paula. 1979. Um novon par vicariante: Mimosa schomburgkii Benth. e Mimosa artemisiana Heringer \& Paula sp. nov. (Floresta Atlántica). XXX Congresso Nacional de Botânica do Brasil. Anais Sociedade Botânica e Brasil 30: 75-82.

Hintze, J. 2001. Number cruncher statistical system (NCSS) 2001. NCSS, LLC. Kaysville, USA. www.ncss.com.

IAWA Committee. 1989. The International Association of Woods Anatomists list of microscopic features for hardwood identification. The International Association of Wood Anatomists Bulletin 10: 219-332.

Jansen, S., P. Baas, P. Gasson, F. Lens y E. Smets. 2004. Variation in xylem structure from tropics to tundra: evidence from vestured pits. Proceedings of the National Academy of Sciences USA 101(23): 8833-8837. DOI: http://dx.doi. org/10.1073/pnas.0402621101

Jansen, S., P. Baas, P. Gasson y E. Smets. 2003. Vestured pits: do they promote safer water transport? International Journal of Plant Sciences 164(3): 405-413. DOI: http://dx.doi. org/10.1086/374369

Johansen, D. A. 1940. Plant Microtechnique. Mc Graw Hill. Nueva York, USA. 523 pp.

Jono, V. 2009. Anatomia ecológica do Lenho e actividade cambial de Roupala rhombifolia (Proteaceae) na Serra do Cipó (MG). Dissertação de mestrado, área Botânica. Instituto de Biociências, Universidade de São Paulo. São Paulo, Brasil. 75 pp. DOI: http://dx.doi.org/10.11606/D.41.2009. tde-13072009-113356

León, H. W. 2001. Anatomía del leño, aspectos ecológicos y filogenia en mangles de Venezuela. Revista Forestal Venezolana 45(2): 191-203.

León, H. W. 2001-2002. Anatomía xilemática del tronco de Pereskia guamacho (Cactaceae) procedente de Mérida-Venezuela. Pittieria 31: 41-49.

León, H. W. 2005. Anatomía ecológica del xilema secundario de un bosque seco tropical de Venezuela. Acta Botánica Venezuelica 28: 257-274.

Lindorf, H. 1994. Eco-anatomical wood features of species from a very dry tropical forest. The International Association of Wood Anatomists Bulletin 15(4): 361-376. DOI: http://dx.doi.org/10.1163/22941932-90001370

Luchi, A. E. 1998. Periodicidade de crescimento em Hymenaea courbaril L. e anatomia ecológica do lenho de espécies de mata ciliar. Tese de doutorado, área Botânica. Instituto de Biociências, Universidade de São Paulo. São Paulo, Brasil. 236 pp.

Maccari, A. y J. N. C. Marchiori. 1994. Estudo anatômico do xilema secundário de Mimosa sparsa Benth. Ciência Florestal 4: 145-155.

Marchiori, J. N. C. 1982. A estrutura do xilema secundário de Mimosa daleoides Benth. (Leguminosae-Mimosoideae). Ciência e Natura 4: 107-113.

Marchiori, J. N. C. 1985. Anatomia da madeira de Mimosa cruenta Benth. (Leguminosae Mimosoideae). Ciência e Natura 7: 73-81. 
Marchiori, J. N. C. 1993. Anatomia da madeira e casca do maricá, Mimosa bimucronata (DC.) Kuntze. Ciência Florestal 3: 85-106.

Marchiori, J. N. C. 1996. Anatomia do xilema secundário de $M i$ mosa incana (Spreng.) Benth. Ciência Florestal 6(1): 53-63.

Marchiori, J. N. C. y B. G. Muñiz. 1997. Estudo anatômico do xilema secundário de Mimosa trachycarpa Benth. Ciência Rural 27(2): 223-228. DOI: http://dx.doi.org/10.1590/ S0103-84781997000200009

Martínez-Bernal, A. y R. Grether. 2006. Mimosa. Flora del Valle de Tehuacán-Cuicatlán 44: 42-108.

Miller, H. 1975. Anatomical characteristics of some woody plants of the Angmassalik district of southeast Greenland. Meddelelser om Grønland 198: 1-30.

Moglia, G. y A. Giménez. 1998. Rasgos anatómicos característicos del hidrosistema de las principales especies arbóreas de la región chaqueña argentina. Investigación Agraria. Sistemas y Recursos Forestales 7: 53-71.

Moglia, J. y C. López. 2001. Tendencias de variación radial del leño en Aspidosperma Quebracho Blanco. Investigación Agraria. Sistemas y Recursos Forestales 10(1): 69-79.

Montaño-Arias, S. A. 2010. Anatomía comparada de la madera de especies arbóreas mexicanas del género Mimosa sección Batocaulon (Leguminosae). Tesis de maestría. Universidad Autónoma Metropolitana, Unidad Iztapalapa. México, D.F., México. 141 pp.

Montaño-Arias, S. A., S. L. Camargo-Ricalde, R. Grether y C. De la Paz Pérez-Olvera. 2011. La madera en función del ambiente: un análisis ecoanatómico. Elementos Ciencia y Cultura 18(83): 19-24.

Montaño-Arias, S. A., S. L. Camargo-Ricalde y C. De la Paz Pérez-Olvera. 2013. Ecoanatomía de los elementos de vaso de la madera de cinco especies del género Mimo$s a$ L. (Leguminosae-Mimosoideae). Botanical Sciences 91(1): 1-10.

Novaes, F., Ch. Callado, M. V. Pereira-Moura y H. R. P. Lima. 2010. Wood anatomy of Mollinedia glabra (Spreng.) Perkins (Monimiaceae) in two restinga vegetation formations at Rio das Ostras, RJ, Brazil. Anais da Academia Brasileira de Ciências 82(4): 915-924. DOI: http://dx.doi. org/10.1590/S001-37652010000400014
Parra, M. J. 2010. Determinación de índices de vulnerabilidad y mesomorfia en especies de laurales de la selva San Eusebio (Mérida, Venezuela). Pittieria 34: 13-22.

Polanco, T. C. y P. D. C. Grande. 2009. Análisis ecoanatómico, evolutivo y comparativo de la madera de 40 especies de dos asociaciones del bosque alto andino colombiano. Colombia Forestal 12: 183-203. DOI: http://dx.doi. org/10.14483/udistrital.jour.colomb.for.2009.1.a13

Rzedowski, J. 1978. Vegetación de México. Limusa. México, D.F., México. 482 pp.

Rzedowski, J. 1991. Diversidad y orígenes de la flora fanerogámica de México. Acta Botanica Mexicana 14: $3-21$.

Särkinen, T. E., J. L. Marcelo-Peña, A. D. Yomona, M. F. Simon, R. T. Pennington y C. E. Hughes. 2011. Underestimated endemic species diversity in the dry inter-Andean valley of the Río Marañón, northern Peru: An example from Mimosa (Leguminosae, Mimosoideae). Taxon 60: 139-150.

Silva, A., Blanco C. y H. Lindorf. 1989. Anatomía de la madera de nueve leguminosas de Venezuela. Acta Botanica Brasilica 2(1): 115-134. DOI: http://dx.doi.org/10.1590/ S0102-33061988000300011

Silva, L. B. D., F. D. A. R. D. Santos, P. Gasson y D. Cutler. 2011. Comparative study of Mimosa ophthalmocentra Mart. ex Benth. and Mimosa tenuiflora (Willd.) Poir. (Fabaceae-Mimosoideae) wood in the caatinga of Northeast Brazil. Acta Botanica Brasilica 25: 301-314. DOI: http://dx.doi.org/10.1590/S010233062011000200006

Simon, M. F., R. Grether, L. P. de Queiroz, T. E. Sarkinen, V. F. Dutra y C. E. Hughes 2011. The evolutionary history of Mimosa (Leguminosae): towards a phylogeny of the sensitive plants. American Journal of Botany 98(7): 12011221. DOI: http://dx.doi.org/10.3732/ ajb.1000520

Sokal, R. y F. Rohlf. 1995. Biometry. Freeman and Company. San Francisco, USA. 887 pp.

Sosa, V. y P. Dávila. 1994. Una evaluación del conocimiento florístico de México. Annals of the Missouri Botanical Garden 81(4): 749-757. DOI: http://dx.doi. org/10.2307/2399919 
Wodzicki, T. J. 2001. Natural factors affecting wood structure. Wood Science and Technology 35: 5-26. DOI: http://dx. doi.org/10.1007/s002260100085

Woodcock, D. W. 1994. Occurrence of woods with a gradation in vessel diameter across the ring. The International Association of Wood Anatomists 15: 377-385. DOI: http:// dx.doi.org/10.1163/22941932-90001371
Zimmermann, M. 1978. Vessel ends and the disruption of water flow in plants. Phytopathology 68: 253-255. DOI: http:// dx.doi.org/10.1094/Phyto-68-253

Zweypfenning, R. C. V. J. 1978. A hypothesis on the function of vestured pits. The International Association of Wood Anatomists Bulletin 1: 3-15. 